\title{
Project Tomo: Toward Atomic-scale Analytical Tomography
}

Thomas Kelly ${ }^{1}$, Rafal Dunin-Borkowski ${ }^{2}$ and Joachim Meyer ${ }^{3}$

${ }^{1}$ Steam Instruments, Inc., Madison, Wisconsin, United States, ${ }^{2}$ Forschungszentrum Juelich, Juelich, Nordrhein-Westfalen, Germany, ${ }^{3}$ Rhineland Westfalia Technical University (RWTH), Aachen, Nordrhein-Westfalen, Germany

Microscopists understand that determining the position and identity of every atom in a structure is an ideal that would be valuable for science and technology. This may be called atomic-scale tomography (AST). Transmission electron microscopy has been an extremely successful microscopy for elucidating the structure of materials and it does provide two-dimensional and some three-dimensional information about atom positions. Some beautiful and challenging work has demonstrated that S/TEM can determine the three-dimensional positions of thousands of atoms in a monatomic [1] or diatomic [2] specimen. However, consider the general case of microstructural characterization of engineering materials on a scale that can be related to the properties. Many different elements are present and information is required on much larger scales. For example, the visible structure in an image displayed in a TEM on a $100 \mathrm{~nm}$ by $100 \mathrm{~nm}$ area of a $100 \mathrm{~nm}$ thick specimen (a common scenario) contains $10^{8}$ atoms. This begs the question whether any microscopy could ever determine the position and identity of every atom in a specimen on the size scale of such microstuctural features of interest. Atom probe tomography determines the position and identity of large numbers of atoms $\left(>10^{8}\right)$ with excellent atom identification through time-of-flight spectrometry but it has two main limitations: a) the current detector technology has $80 \%$ detection efficiency and b) reconstruction errors limit the spatial positioning precision to worse than atomic separation distances in most cases. A project to address these two limitations and deliver atomic-scale tomography [3], Project Tomo, was proposed in 2015 in Germany by the authors and was funded in 2019. The equipment manufacturers for this project have been chosen and design work is underway. This project will address the first limitation by developing a position-sensitive detector (Steam Instruments, Inc.) based on superconducting detector technology to achieve $100 \%$ detection efficiency. The second limitation will be addressed by building the atom probe (CAMECA Instruments, Inc.) inside the objective lens of a highquality TEM (ThermoFisher Scientific) to provide information needed to correct reconstruction as described below. This combination takes two highly complementary techniques and uses them synergistically to overcome the limitations of each. Superconducting ion detection has taken many forms and shown great potential but heretofore, this technology has been too slow, had too small of an active detection area, and was too complex for atom probe tomography. In a project seeking to overcome these limitations and deliver 100\% detection efficiency in a large-area detector, McDermott and Suttle [4] devised a superconducting delay line detector. Suttle [5] showed that this concept is feasible and Steam Instruments is developing the technology for commercial application. The project seeks to deliver a 60 $\mathrm{mm}$ by $60 \mathrm{~mm}$ detector capable of $>10^{7}$ ions per second with " $100 \%$ " [6] detection efficiency. Correcting reconstruction errors in atom probe tomography requires information that is lacking in stand-alone atom probes. The errors arise because standard reconstruction algorithms assume a hemispherical end cap on the specimen which is projected onto the planar detector. When the endcap is not hemispherical, the reconstruction is not correct which is most of the time. The solution is to determine the apex shape and electric field distribution surrounding the atom probe specimen well enough to back-compute the ion trajectories from the detector to the specimen. The problem is thus a technology limitation not a physics limitation. By building the atom probe inside of a TEM, with the specimen situated at the usual eucentric position in the column, the specimen endcap shape may be determined with high precision. Furthermore, 
the three-dimensional electric field distribution about the specimen may also be determined in a TEM using off-axis holographic methods [7,8] or a differential phase contrast technique [9]. This project will thus introduce an atom probe into the objective lens of a $\mathrm{C}_{\mathrm{s}}$-corrected scanning transmission electron microscope (S/TEM), Figure 1, which illustrates the nominal configuration with a specimen cartridge in the objective lens region. The electron column will be a $300 \mathrm{kV}$ S/TEM equipped with electron tomography, electron energy-loss and/or energy dispersive X-ray spectroscopy, and phase contrast techniques for electric field mapping. Because the magnetic field of the objective lens alters the trajectories of ions in the atom probe, this column will be designed with a magnetic-field-free mode while retaining atomic spatial resolution when operating the TEM. A major technical challenge will be to develop a specimen stage that can achieve atomic spatial resolution with a high voltage $(15 \mathrm{kV})$ applied to a cryogenic $(<20 \mathrm{~K})$ needle-shaped specimen all in ultrahigh vacuum (UHV). UHV and cryogenic specimen temperatures are required for operation of the atom probe. In order to record high quality tomograms, the specimen stage will be capable of $\pm 90^{\circ}$ rotation about the long axis of the needle-shaped specimen. The atom probe will be laser-pulsed and have a superconducting delay-line (SCDL) detector for the ions. Because the instrument will have a full complement of analytical detectors, the atomic-scale tomography (AST) data will be complemented by atomic-scale analytical information and the result will be atomicscale analytical tomography (ASAT). Project Tomo is expected to take over three years to produce a fully operational instrument. By summer of 2024, we hope to be recording ASAT data and begin ushering in a new phase for microscopy at the atomic scale [11]. 


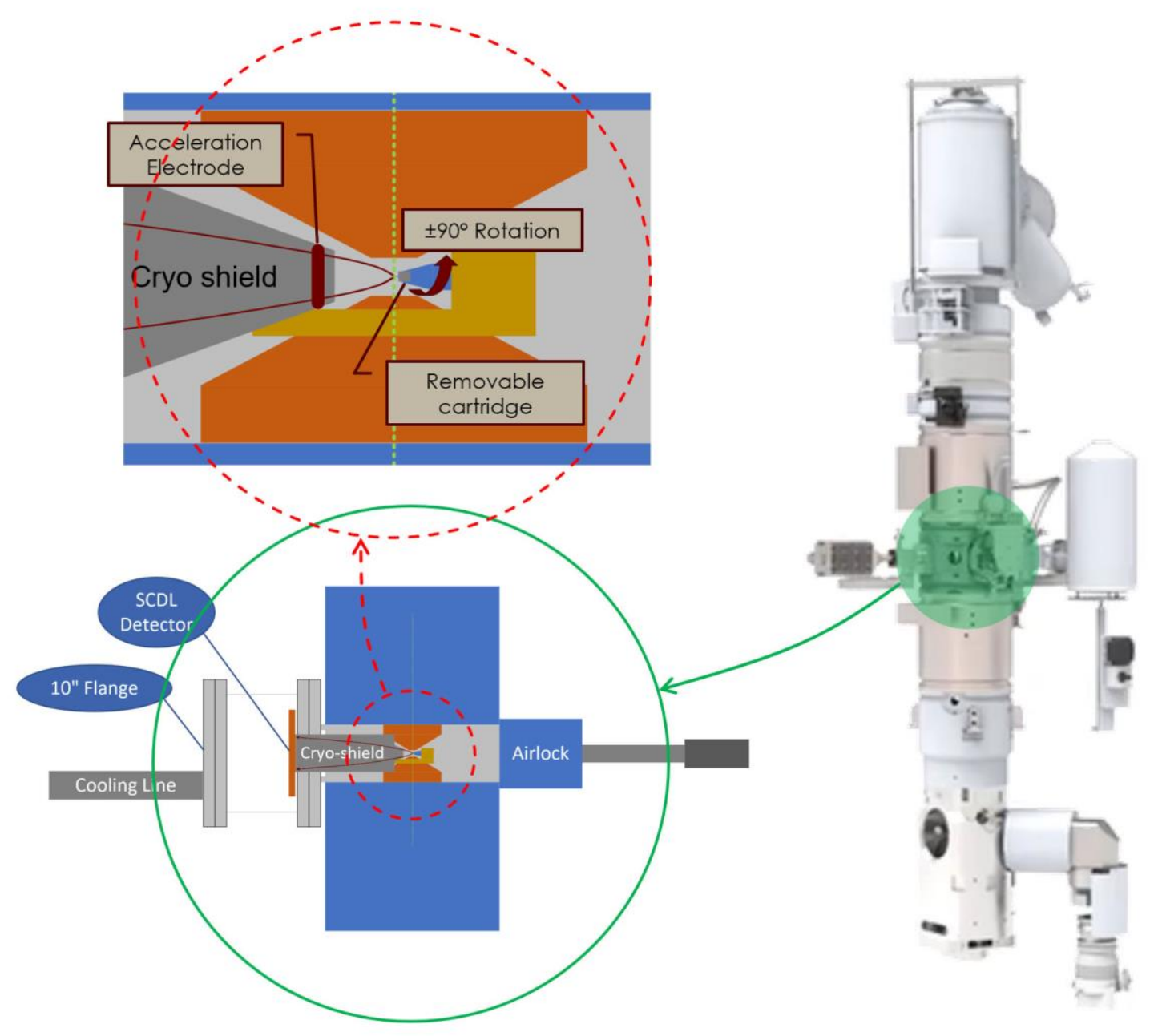

Figure 1. Solid model of the Spectra 300 S/TEM electron column from ThermoFisher Scientific [10] on the right, a schematic of the objective lens region (lower left) and a schematic of the pole piece region of Project Tomo (upper left).

\section{References}

1. Xu R, Chen C-C, Wu L, Scott MC, Theis W, Ophus C, Bartels M, Yang Y, Ramezani-Dakhel H, Sawaya MR, Heinz H, Marks LD, Ercius P, Miao J (2015) Three-dimensional coordinates of individual atoms in materials revealed by electron tomography. Nat Mater.

2. Yang Y, Chen C-C, Scott MC, Ophus C, Xu R, Pryor A, Wu L, Sun F, Theis W, Zhou J, Eisenbach M, Kent PRC, Sabirianov RF, Zeng H, Ercius P, Miao J (2017) Deciphering chemical order/disorder and material properties at the single-atom level. Nature 542:75-79.

3. Kelly TF, Miller MK, Rajan K, Ringer SP (2013) Atomic-Scale Tomography: a 2020 Vision. Microsc Microanal 19:652-664

4. McDermott RF, Suttle JR (2015) US Patent: System and Method for Characterizing Ions Using a Superconducting Transmission Line Detector. US Patent 9490112

5. Suttle J (2018) A Superconducting Ion Detector. Ph.D., The University of Wisconsin - Madison

6. We put $100 \%$ in quotation marks because all real experiments have noise and other limitations. We expect to approach the ideal of $100 \%$ with detection efficiencies that may be $99.9 \%$, for example. 
7. Migunov V, London A, Farle M and Dunin-Borkowski R E (2015) Model-independent measurement of the charge density in an Fe atom probe needle using off-axis electron holography without mean inner potential effects. J. Appl. Phys. 117: 134301.

8. Zheng F, Caron J, Migunov V, Beleggia M, Pozzi G and Dunin-Borkowski R E (2020) Charge density measurement in nanoscale materials using off-axis electron holography. Journal of Electron Spectroscopy and Related Phenomena, in press.

9. Wu M, Tafel A, Hommelhoff P and Spiecker E (2019) Determination of 3D electrostatic field at an electron nano-emitter. Appl. Phys. Lett. 114: 013101.

10. https://www.thermofisher.com/us/en/home/industrial/electron-microscopy/electron-microscopyinstruments-workflow-solutions/spectra-300-s-tem.html

11. Project Tomo is funded as part of the Ernst Ruska-Centre 2.0 project, which is funded by the federal and state ministries in Germany and the Helmholtz Association. 